\title{
Forma Urbana e Inclusione Sociale. Una Discussione Intorno al Concetto di Diritto alla Città
}

\author{
Manuela De Vincenzi ${ }^{1}$
}

\begin{abstract}
The proposed topic concerns, in general, the decision-making process in urban planning in relation to its ideological implications, freedom of choice and the fight against urban poverty. In particular, this study focuses on ideal urban model design, and its subsequent export to different contexts, as a measure of development and reduction of economic and social inequality and marginalization of a certain part of the population. The case studies presented will be the following: the leisure city project "Tróia, garden-city" (1970) and the social housing project "Zona J" (1980). Even though they had different purposes (one was meant to be a middle class and touristic neighborhood, while the other was thought to be a neighborhood for poor people), both have the same formal project design. On one hand, Tróia continues representing a modern and quality touristic destination, "Zona J", on the other hand, has become the emblem of degradation. What was initially meant to be a requalification and urban integration project has eventually become a further element of stigmatization. Through comparison between original intentions, what was actually built and their effective use of spaces, we tried to answer the questions that underlie this work: are there universally valid solutions? What makes the same project a reference work in a given context and the emblem of environmental and social degradation in another? Who are the individuals who take these decisions?
\end{abstract}

Keyword: Shape; Identity; Right to the City; Urban design

\section{Il Progetto Urbano Come Prodotto da Esportazione}

La continua crescita dei grandi centri urbani, l'espansione degli stessi verso le campagne, l'aumento dei contrasti tra centro e periferia, hanno messo in crisi l'utopia modernista di progresso, libertà e uguaglianza che, ad oggi, rimane disattesa. A ricordarcelo sono le periferie di tutto il mondo che, nonostante i buoni propositi iniziali, continuano ad aumentare, a vivere situazioni di isolamento e degrado (Amin, 2012; Bauman, 2013; Secchi, 2011) .

In questo contesto, però, non si tratta di indagare il fenomeno dell'aumento delle periferie in sé, si tratta, invece, di porre l'attenzione su alcune delle idee più forti e emblematiche della modernità legate al concetto di giustizia sociale o, per meglio dire, alla riduzione delle disuguaglianze.

Il focus di questo lavoro si concentra principalmente sul processo decisionale all'interno del progetto urbano. Nello specifico sarà messo in discussione il fenomeno di riproduzione di concetti architettonici, modelli formali, in contesti ambientali diversi.

Una pratica di "importazione ed esportazione" di modelli di urbani (Nasr, Volait, 2003) che ha cominciato ad essere particolarmente visibile a partire dall'industrializzazione delle città e dei problemi legati all'alloggiamento delle masse che arrivavano nei grandi centri urbani in cerca di lavoro e migliori condizioni di vita.

$\grave{E}$ in questo contesto, tra la seconda metà del XIX e inizio del XX secolo, che 
l'urbanistica si afferma come disciplina autonoma, con i suoi strumenti, motivazioni e ideologie (Calabi, 2008).

Con l'avvento della modernità si fa strada un'idea di architettura e, più in generale, di urbanistica, considerata come un linguaggio fisico capace di influenzare le persone in termini di gusto, etica e comportamento. Questa nuova visione del ruolo dell'architettura porterà a diverse sperimentazioni e concetti di abitare (modelli) che, a partire da questo momento, si propagheranno in Europa e nel resto del mondo.

Più avanti saranno presentati due casi di studio ("Troia, Cidade Jardim" e "Zona J") che presentano lo stesso concetto architettonico ma una destinazione d'uso, un contesto socio-economico e geografico, completamente diversi.

Di questo complesso fenomeno, in questo studio, saranno presi in considerazione due aspetti: da un lato, la dimensione ideologica della forma urbana e architettonica, quindi il processo decisionale e $\mathrm{i}$ valori che stanno alla base della realizzazione fisica dell'ambiente e, dall'altro lato, l'interpretazione e l'uso da parte degli abitanti. L'obiettivo, come già accennato, è quello di indagare se e come, le forme, possono incidere sul nostro comportamento ma, soprattutto, se esistono soluzioni universalmente valide che si possano considerare utili ai fini di una maggiore inclusione e riduzione della povertà urbana.

\section{La Forma tra Ideologia e Affermazione Identitaria}

La forma quindi, anche quella architettonica, è analizzata nella sua vertente comunicativa, simbolica, oltre che funzionale. In questo senso, si può considerare la forma urbana come una reificazione di determinate concezioni del mondo e, per questo, può essere utilizzata coscientemente o incoscientemente come strumento coercitivo da parte del gruppo dominante.

Si possono riconoscere almeno due dimensioni comunicative dell'architettura (come di qualunque altro artefatto): una denotativa, che corrisponde a un primo livello di interpretazione e coincide con la funzione di un determinato prodotto. Nel caso di un edificio, per esempio, la sua funzione primaria è quella di "riparo", di "essere abitato". L'altra dimensione, quella connotativa, corrisponde al modo in cui la forma viene concepita. Tornando al nostro esempio, la dimensione connotativa è quella che risulta dalle scelte formali adottate, la disposizione e l'organizzazione dello spazio. Aspetti, questi, che portano con sé significati strettamente legati a specifiche concezioni di abitare, in altri termini trasmettono "lideologia globale che ha presieduto l'operazione dell'architetto" (Eco, 1968:570).

È esattamente in questo secondo aspetto, infatti, che possiamo collocare la dimensione ideologica o mitologica di un progetto, ricordando, al contempo, che "il mito non può essere in nessun modo un oggetto, un concetto o una idea; ̀̀ un modo di significare, una forma" (Barthes, 1957:11).

A questo punto va fatta una distinzione tra l'esigenza naturale di ogni essere umano di affermare la propria identità, di essere riconosciuto e la volontà di imporre un unico modello, quello che il gruppo dominante reputa "giusto" o migliore.

Nell'ambito delle scienze sociali, i primi studi sulla relazione tra forma e funzione come risultato di un processo culturale furono sviluppati da Mauss e Durkheim, nel 1901. Il 
loro studio rappresenta una critica all'interpretazione geografico-tecnologica dell'habitat, dimostrando l'importanza del legame tra forma e organizzazione della vita sociale (Mauss, Durkheim apud La Cecla, 1993).

Cinquant'anni più tardi, Levi-Strauss torna sullo stesso tema, in Tristi Tropici, l'autore racconta di come i Salesiani, per convertire la tribù dei Bororo che abitavano la zona dell'Amazzonia, cominciarono proprio dalla trasformazione fisica del loro ambiente:

I missionari salesiani della regione del Rio das Graças si accorsero da subito che il modo più sicuro per convertire i Bororo consisteva nel fargli abbandonare il loro il villaggio e trasferirli in un altro dove le case erano disposte in file parallele 0 , la distribuzione circolare delle capanne era di estrema importanza per la vita sociale e le pratiche di culto della tribù (Lévi-Strauss, 1966:232)

I bororo si trovarono, così, disorientati nel nuovo ambiente senza riuscire a riprodurre le stesse pratiche quotidiane e legami sociali che fino ad allora avevano caratterizzato la loro comunità.

In questo senso, la forma costruita, oltre che manifestazione identitaria può farsi mezzo di coercizione, assumendo i tratti tipici dell'ideologia.

A tal proposito, Lefebvre (1970) riferisce che "I'urbanistica come dottrina e, quindi, come ideologia, interpreta le conoscenze specializzate, giustificandone le applicazioni, elevandole (per estrapolazione) a una generalità mal fondata o mal legittimata” (Lefebvre, 1970:104).

Sempre negli stessi anni, Blumer parla di "interazionismo simbolico" evidenzioando, appunto, l'importanza del processo interpretativo nelle relazioni tra esseri umani e tra persone e oggetti. Per l'autore, il modo di agire verso cose e persone partirebbe dal significato che questi hanno per l'individuo agente (Blumer, 1969).

\section{Metodologia}

La finalità di questo lavoro è quella di mettere in discussione la riproduzione di modelli architettonici come linguaggi universali e, per questo, esportabili in diversi contesti. Più in generale si vuole indagare le reali "responsabilità" della forma intesa come strumento capace di inibire o amplificare le problematiche legate alla marginalità e all'inserzione sociale dei gruppi sfavoriti.

Il caso proposto in queste pagine è particolarmente emblematico poiché, lo stesso progetto architettonico, è stato utilizzato per la realizzazione di due quartieri completamente diversi, non solo in termini di collocazione geografica ma anche come destinazione d'uso e tipologia di abitanti. Proprio per questo, dal punto di vista metodologico, risulta essere particolarmente complicata una comparazione diretta tra le due situazioni. Si è optato, invece, per una analisi indipendente dei due casi con l'obiettivo di intercettare e comprendere le logiche di appropriazione e utilizzo degli spazi nel corso del tempo, in un caso e nell'altro.

In un primo momento si è proceduto con l'analisi di documenti ufficiali relativi ai progetti per comprenderne le intenzioni generali, cosa effettivamente è stato costruito e perché. Oltre alla letteratura esistente sui casi di studio, sono state utilizzate mappe, memorie descrittive, statistiche e ricerche effettuate sulla composizione sociale delle zone interessate. Si è provveduto, poi, ad individuare gli aspetti formali di particolare interesse per questo lavoro. In altre parole, esistono degli elementi costitutivi comuni ai due casi 
pensati con il proposito di incidere sullo sviluppo sociale ed economico di queste zone. In questo senso, sono state individuate le seguenti referenze: Forma urbana; Volumetria; Funzioni e usi degli edifici; Sistema di circolazione e spazi intermedi; Relazione con lo spazio pubblico. In un secondo momento si è proceduto con le interviste indirizzate principalmente ai funzionari pubblici coinvolti nei progetti, l'architetto (comune a entrambi) e i rappresentanti delle associazioni coinvolte (quelle dei residenti e gestione locale dei servizi), incontri che si sono spesso svolti sul luogo. Infine, con l'osservazione diretta (basata sugli elementi formali individuati in precedenza), è stato possibile conoscere i comportamenti degli abitanti e il tipo di relazioni che hanno luogo nei due contesti. In sintesi, considerando che l'oggetto di studio ruota intorno alla forma costruita dell'ambiente in quanto reificazione di concetti di abitare, è stato seguito uno schema di analisi di "concezione - costruzione - utilizzazione" (quali propositi iniziali, cosa è stato realmente costruito e come è stato interpretato e utilizzato da parte dei destinatari).

\section{4. "Troia, Cidade Jardim": il paesaggio come prodotto competitivo.}

"Troia, Cidade Jardim" (foto 1) è un progetto di iniziativa privata sorto nei primi anni settanta e concepito dall'architetto Francisco Conceição da Silva. Occupa un'area di circa 40 ha a nord della penisola di Troia, localizzata a sud di Lisbona. Fu ideato come risposta alle nuove esigenze della classe media portoghese (che in questi anni, con le nuove conquiste sindacali, come il diritto alle ferie pagate, migliorarono la loro condizione economica) e, più in generale, alla classe media europea. In questo senso, lo sviluppo del turismo verso il mare (incentivato anche attraverso la propaganda mediatica) e l'abbassamento dei costi legati al viaggio, portarono a quello che conosciamo come turismo di massa. Tale circostanza ha favorito la nascita di città (o parti della stessa) destinate al turismo, ambito nel quale il Portogallo, per esempio, era ancora incipiente. "Troia, Cidade Jardim" si può considerare a tutti gli effetti la prima città turisticoresidenziale, completamente costruita dalla radice, del Portogallo (Silva; 1971; Leite, 2007).

Con la caduta del governo di Salazar nel 1974, la crisi del petrolio del 1975 e l'ondata di "ritornati" (retornados) dalle colonie portoghesi, il progetto fu interrotto e lasciato in stato di abbandono per oltre vent'anni.

Solo sul finire degli anni novanta vennero eseguiti alcuni lavori di implementazione del progetto, tuttavia, senza portare alla conclusione dello stesso.

Nonostante, oggi, esista solo una piccola parte del progetto iniziale e pur non avendo raggiunto quell'ideale di vita urbana immaginato, Troia continua a funzionare come una delle principali località turistiche in Portogallo e la grande maggioranza delle abitazioni costruite risultano occupate come seconde case.

È importante sottolineare che, in questo caso, non si tratta semplicemente di una città costruita ex novo, ma della creazione di un vero e proprio territorio del turismo che bisognava immaginare e disegnare (Silva, 1970). In questo senso, l'architetto sosteneva che non fosse sufficiente costruire nuove abitazioni o hotel seppur immersi in luoghi da favola, bisognava dare la possibilità al turista di vivere esperienze uniche e a portata di mano. Offrire le attività e servizi che si possono trovare in una città ma in un luogo 
rilassante, lontano dal traffico e dallo stress urbano (Silva, 1970).

Dal punto di vista morfologico, il progetto è il risultato di una sintesi tra alcune idee tipicamente moderniste: la città giardino e il funzionalismo. Le strutture abitative, infatti, sono composte da blocchi di volume diverso (tra i 4 e i 9 piani più le torri che arrivano fino a 16 piani) a causa delle interruzioni furono costruiti solo i primi 7 blocchi di 4 piani disposti in modo da formare dei quadrilateri intorno a giardini pubblici provvisti di attrezzature per lo sport e il tempo libero. I vari edifici, inoltre, sono collegati tra loro per mezzo di gallerie e passerelle, all'altezza del primo piano, in modo da poter percorrere a piedi l'intera zona edificata, separando così il traffico delle automobili da quello dei pedoni (foto 2-4).

Alla densità abitativa degli edifici, si contrappone una grande quantità di giardini e spazi pubblici attrezzati, servizi di vario genere con l'obiettivo, da un lato, di abbassare i costi delle unità abitative, dall'altro, di offrire soluzioni di alta qualità sia in termini strutturali che ambientali.

Possiamo considerare "Troia, Cidade Jardim", un progetto utopistico, più che ideologico, volto a creare un concetto di abitare nuovo che potesse integrare il turismo di massa con la parte più residenziale, in un'area indipendente, simile, a tutti gli effetti, ad una città.

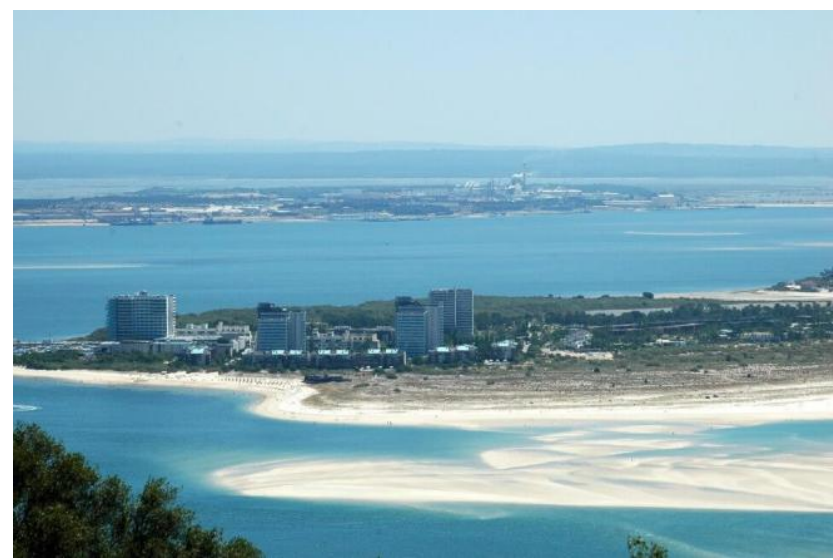

Foto 1. Vista aerea di "Troia, Cidade Jardim. CCTalents, 2016

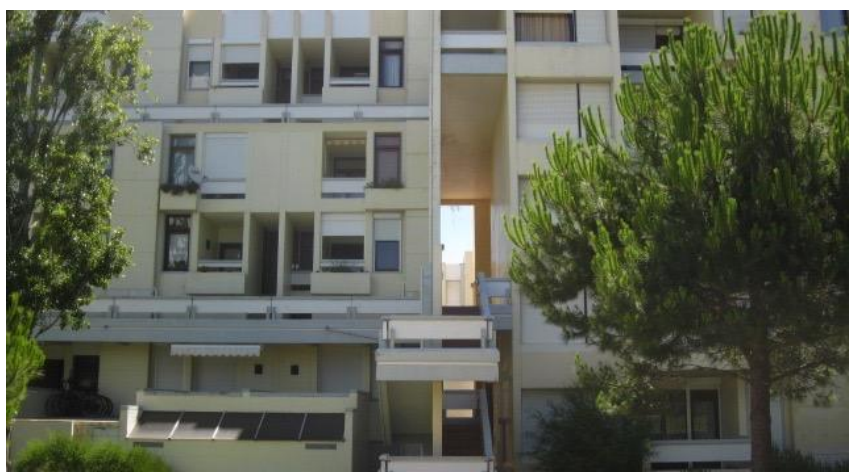

Foto 2. Vista di una facciata e sistema di gallerie. Foto realizzata dall'autrice nel 2015 

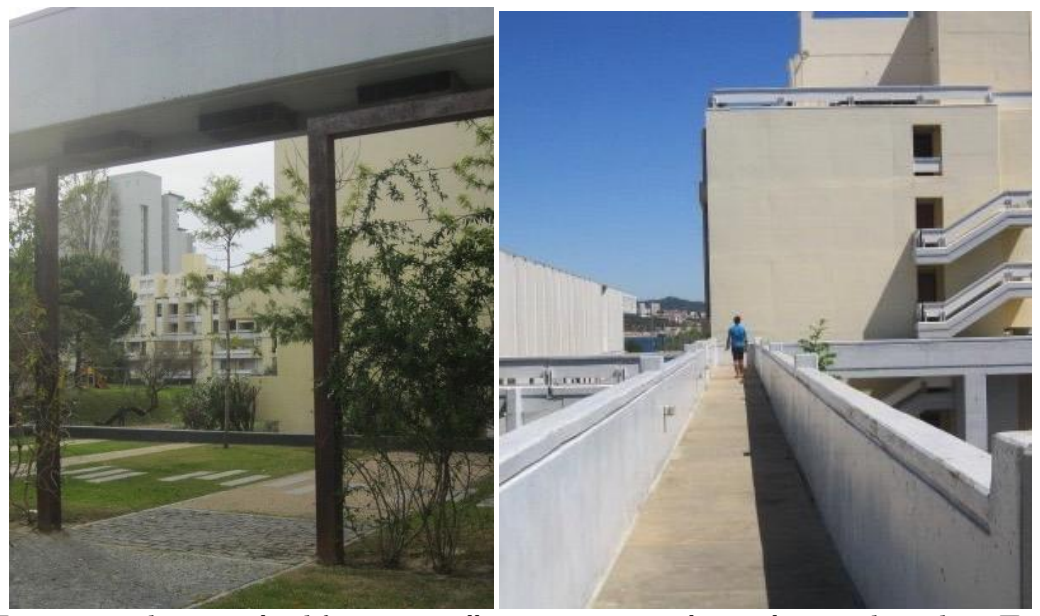

Foto 3-4. Entrata giardino tra gli edifici e passerelle soprastanti per la circolazione dei pedoni. Foto realizzata dall'autrice nel 2015

\section{5. "Zona J": I1 Riscatto Mancato di Una delle Zone più Stigmatizzate di Lisbona}

Sorto negli anni ottanta nella periferia orientale di Lisbona e ideato da uno degli ex collaboratori dell'Atelier di Conceição Silva, l'architetto Tomás Taveira, la "Zona J" (foto 5) è un progetto di iniziativa pubblica destinato ad accogliere parte dei retornados dalle antiche colonie portoghesi e, in generale, la parte della popolazione con scarse risorse economiche, costretta a vivere in condizioni fortemente precarie.

L'obiettivo del Piano di Urbanizzazione era quello di "sviluppare una struttura urbana plurifunzionale, socialmente diversificata e integrata nel congiunto della cittă” (GTH, 1964:141).

Oltre a migliorare le condizioni abitative di migliaia di persone (circa 9000) che in questa zona vivevano in alloggi di fortuna, l'idea, quindi, era quella di riqualificare l'intera area e annetterla al resto del tessuto urbano.

Con questo proposito, nel 1976 il progetto di Tomás Taveira fu scelto dalla Camera Municipale di Lisbona perché considerato la migliore soluzione architettonica per il nuovo quartiere. Di fatto, però, lo schema generale della struttura fisica già esisteva ed era stato elaborato dall'allora chiamato Gabinete Tecnico de Habitação (GTH) della Camera Municipale di Lisbona (foto 6). A tal proposito, è interessante notare come alcuni dei requisiti formali del piano fossero già stati decisi a priori come, ad esempio, il volume e tipologia degli edifici, che dovevano essere costruiti con il sistema delle gallerie in modo da collegare i diversi blocchi e separare i pedoni dal traffico stradale:

"i percorsi per $i$ pedoni stanno alla base della vivificazione della zona e si sviluppano utilizzando una sequenza di gallerie che vanno dagli elementi estremi, fino alla galleria comunitaria che struttura la fascia centrale.

Si cerca, in questo modo, di creare un terzo spazio, intermedio tra lo spazio interiore e lo spazio esteriore, che permetta a un abitante della zona di percorrerla nella sua totalità, sempre riparati da costruzioni e, quindi, in condizioni speciali di confort e sicurez:a" (GTH, 1975:134)

Il progetto architettonico proposto da Taveira è, come da lui stesso sostenuto, basato sul modello di "Troia, Cidade Jardim" (Taveira, 2015), al quale aveva lavorato insieme a 
Conceição Silva, riproducendo esattamente la stessa concezione formale degli spazi. Progetto che, d'altronde, si adattava molto bene alle richieste della Camera (foto 7-8).

I propositi iniziali, però, non si concretizzarono, a partire dall'integrazione della "Zona J" con il resto della città formale, ancora oggi completamente isolata dal resto del tessuto urbano. Inoltre, gli spazi pensati come "propulsori di vita urbana intensa" (GTH, 1975:71), sia in termini economici che sociali, finirono, al contrario, per incentivare comportamenti trasgressivi. Tra gli esempi più emblematici c'è sicuramente la galleria centrale (che attraversa l'intera area), pensata come cuore commerciale del quartiere e, invece, diventata famosa come "corridoio della morte" (foto 9) a causa delle numerosi morti legate alla droga, violenza tra gang, attività illecite di vario genere che si concentravano in luoghi come questo a causa della poca visibilità. Per questi motivi, a partire dal 2009, la galleria e altri interstizi simili che nel corso degli anni avevano contribuito alla creazione di stereotipi fortemente negativi verso gli abitanti della "Zona J", furono demoliti. Gli abitanti, sia per motivi di sicurezza che per motivi di decoro urbano, cominciarono a occupare abusivamente gli spazi comuni, spesso in stato di abbandono, intorno alle loro abitazioni, recintandoli, per impedirne il passaggio a persone sconosciute e arredandoli, come vere e proprie estensioni delle loro abitazioni (foto 10).

Se da un lato, i residenti riconoscono il miglioramento delle condizioni abitative per quanto riguarda gli alloggi, dall'altro lato, lamentano la perdita degli antichi legami sociali e, soprattutto, un certo modo di vivere il quartiere (Santos, 2015). Proprio quel "terzo spazio" così fortemente voluto dai decisori politici, si è rivelato un grande ostacolo alla vivificazione del quartiere. Creando una sorta di divisione tra lo spazio pubblico e quello privato, le persone si sono trovate a passare molto più tempo in casa di quanto non facessero anteriormente (Santos, 2015).

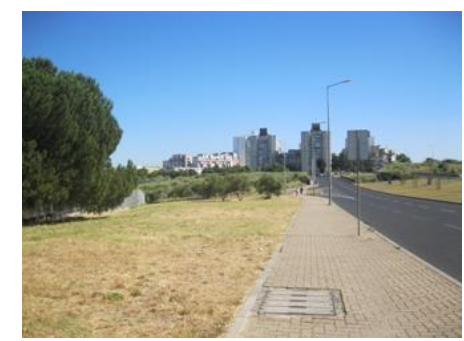

Foto 5. Vista della "Zona J". Foto realizzata dall'autrice nel 2016

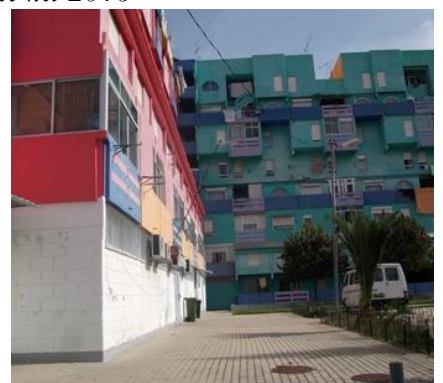

Foto 7. Vista parziale degli edifici. Foto realizzata dall'autrice nel 2014.

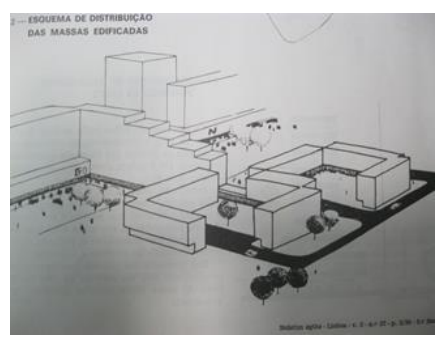

Foto 6. Schema struttura "Zona J". GTH, 1974

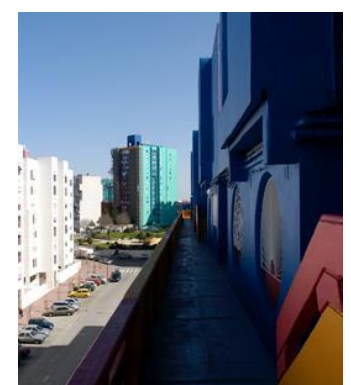

Foto 8. Vista parziale dei corridoi-passerelle che collegano gli edifici. Foto concessa dall'architetto Tomás Taveira. 


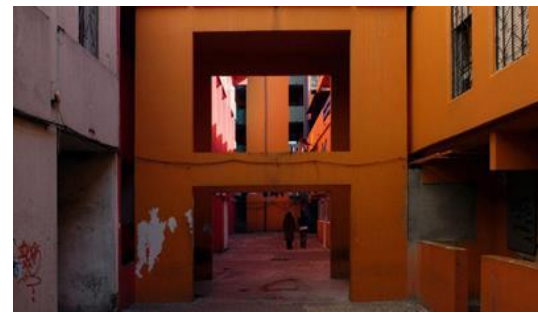

Foto 9. Vista parziale del "corridoio della morte". Foto concessa dall'Architetto Tómas Taveira.

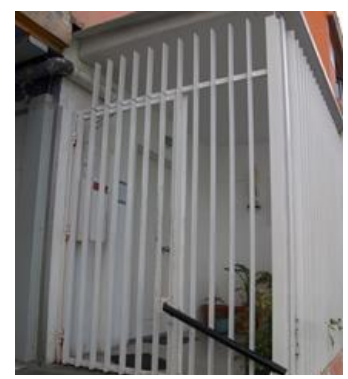

Foto 10. Spario comune occupato e recintato abusivamente. Foto realizzata dall'autrice nel 2014.

\section{Risultati}

Sia nel caso di "Troia, Cidade Jardim" che nel caso della "Zona J", i propositi iniziali previsti dal Piano non furono completamente realizzati. Nel primo caso, però, nonostante il progetto sia stato interrotto, senza vedere mai concretizzata quell'idea di vita urbana immaginata, "Troia, Cidade Jardim" continua a funzionare come località turistica. Inoltre, va sottolineato come questo progetto, di natura privata, sia sorto per rispondere alle richieste di un target ben definito e che, di fatto, ha deciso di investire in questo luogo.

Nel caso della "Zona J" si può considerare come unico obiettivo raggiunto il miglioramento strutturale delle singole unità abitative. Nonostante sia fornito di tutti i servizi basici, l'area rimane fortemente marginalizzata rispetto al resto della città, gli abitanti tendono a uscire poco dal quartiere e a sentirsi stigmatizzati (Santos, 2015). Dichiarano, inoltre, che anche se avessero la possibilità di cambiare quartiere, cambierebbero solo per un altro dello stesso statuto (un altro quartiere di edilizia pubblica), perché qui, dicono, sono “tra uguali” (Santos, 2015). Questo aspetto si lega ad un altro problema particolarmente sentito dalle autorità locali: la forte dipendenza degli abitanti nei confronti dello Stato. I funzionari intervistati, lamentano il disinteresse dei residenti nei confronti della manutenzione dell'edificato e degli spazi comuni ${ }^{1}$.

Dal punto di vista della concezione formale, se "Troia, Cidade Jardim" può essere considerato un progetto utopistico perché mira alla realizzazione di un luogo nuovo, quello della "Zona J" è chiaramente una concezione ideologica e moralista perché riproduzione "alla maniera" di un'idea precisa, già esistente e considerata in termini assoluti la migliore.

Infine, vale la pena notare la forte differenza nell'uso degli spazi edificati in un caso e nell'altro. Nel primo caso, come facilmente intuibile, non si manifestano problemi di vicinato e gli spazi sono curati e tenuti in ordine tramite l'associazione dei proprietari. La partecipazione degli inquilini alle questioni riguardanti il territorio è molto forte al punto da potersi considerare quasi una lobby nella difesa dei loro interessi ${ }^{2}$. Situazione inversa,

\footnotetext{
${ }^{1}$ Intervista realizzata dall'autrice ai tecnici comunali responsabili per la "Zona J”. Lisbona, settembre 2017

2 Intervista realizzata dall'autrice al presidente dell'associazione dei proprietari di “Troia, Cidade Jardim”, novembre 2017.
} 
nel caso della "Zona J" dove esistono molti problemi tra inquilini, legati soprattutto alla manutenzione degli edifici, alla sicurezza e degrado ambientale ${ }^{3}$. Anche in questo caso, come riportato dai funzionari comunali, esistono delle differenze tra chi è proprietario e chi invece è affittuario, esiste una maggior partecipazione dei primi alle decisioni che riguardano il quartiere. Questo aspetto va a sottolineare il peso che ha la possibilità di scelta, oltre che lo status socio-economico, sul grado di partecipazione e inclusione dei singoli individui nella vita comunitaria.

\section{Conclusioni}

Tornando alle domande che stanno alla base di questo lavoro, è difficile pensare a concetti di abitare che siano universalmente validi e che possano essere intesi come modelli "ideali" ai quali ispirarsi per promuovere inclusione e partecipazione. Nello stesso modo, non si possono considerare le forme sbagliate in termini assoluti.

Ciò che, invece, si può considerare universale è l'importanza che qualunque popolo, gruppo sociale e individuo attribuisce alla forma, all'babitat, in quanto, come si è inizialmente specificato, costruzione simbolica del proprio ambiente. La forma riveste un ruolo importante perché rappresentazione fisica del nostro modo di stare al mondo e di essere riconosciuti. A questo proposito, però, si è fatta distinzione tra la mera rappresentazione identitaria (da intendersi come libertà di scelta, di stare al mondo) e l'imposizione dall'alto, la coercizione. La potenza comunicativa dell'arte e dell'architettura è storicamente riconosciuta, spesso, infatti, sono state messe al servizio del potere dominante col fine di promuovere questa o quella ideologia.

Alla luce di quanto detto e se consideriamo vero quanto già sostenuto da Dorfles (2010) che "la libertà di scelta è la dimensione più vitale dell'essere umano" (Dorfles, 2010:134), allora sarebbe certamente più efficace pensare a un modo per far si che queste scelte diventino realtà per tutti. Diventa quindi indispensabile investire in politiche pubbliche in grado di fornire possibilità e meno nella politica del prêt-à-porter.

\section{References}

Amin A., (2012). "Discorso di apertura dell'anno accademico all'Università di Cambridge", in The post-Urban Age: toward a new form of settlement, Boundaries magazine, Roma.

Arquitectura (1971) "Entrevista com o Arquiteto Francisco Conceição Silva", Arquitectura , 3. ${ }^{a}$ série, n. ${ }^{\circ}$ 120, Março/Abril, 1971, pp. 42-46.

Barthes, R. (1975). Mitologias. Versão traduzida por José Augusto Seabra. Lisboa: Edições 70.

Bauman Z., (2013). Communitas. Uguali e diversi nella società liquida. Trad. di Carlo Bordoni. Reggio Emilia: Aliberti Editore.

Blumer, H. (1969), Symbolic Interactionism. Perspective and Method. Englewood Cliffs, Prentice-Hall.

Calabi D., (2008). Storia dell'urbanistica europea. Milano, Mondadori.

Dias, F. S. e Carvalho, J. A. L. (1974) "Plano de Urbanização de Chelas - Zona J", Boletim do Gabinete Técnico da Habitação 6, n. ${ }^{\circ} 35,2^{\circ}$ semestre, pp.3-30, CML, Lisboa.

Dorfles G., (2010). Dal significato alle scelte. Roma, Castelvecchi.

Eco, U. (1968) "A Estrutura Ausente", Teoria e Crítica de Arquitetura - Seculo XX, Casal de Cambra: Caleidoscópio, 2010

\footnotetext{
${ }^{3}$ Intervista realizzata dall'autrice ai tecnici comunali responsabili per la "Zona J". Lisbona, settembre 2017
} 
GTH (1964), Principal Legislação da Habitação Económica Interessando à Cidade de Lisboa - de 1912.07.26 a 1964.12.28, Boletim do Gabinete Técnico da Habitação 1, n.o 3, Nov.-Dez. 1964, pp.140-172.

GTH (1962), Plano de Urbanização de Chelas - Plano-base, Boletim do GTH, Lisboa

GTH (1974). Plano Urbanístico de Chelas - Zona J, Boletim do Gabinete Técnico de Habitação, Câmara Municipal de Lisboa

GTH (1975) "Concurso das empreitadas conceção - construção da Zona J", Boletim do Gabinete Técnico de Habitação da Câmara Municipal de Lisboa, n. ${ }^{\circ}$ 28, pp. 67-81

GTH (1975) "Conceção dos edifícios - Zona J", Boletim do Gabinete Técnico de Habitação da Câmara Municipal de Lisboa, n. ${ }^{\circ}$ 29, pp. 153-189

La Cecla F., (1993). Mente Locale. Per un'antropologia dell'abitare. Milano, Elèuthera.

Lefebvre, H. (1968). Diritto alla città. Trad. de Cesare Bairati (1970), Padova, Marsilio Editori.

Leite I., (2007). Atelier Conceição Silva: Território e turismo, in Jornal Arquitectos 227, pp. 30 - 31.

Lévi-Strauss C. (1966). Tristi Tropici. Milano: Il Saggiatore

Nasr J., Volait M., (2003). Urbanism: Imported or Exported?. New York, Wiley.

Sampò L., (2012). The post-Urban Age: toward a new form of settlement in Boundaries magazine. Roma

Santos, M. (2015). Tão Perto e tão longe. Satisfação residencial e participação cívica nos bairros municipais de Lisboa. Casal de Cambra: Caleidoscópio

Secchi B., (2011). La città del ventesimo secolo. Bari, Editori Laterza

Silva F. C., (1972). Que turismo para Portugal?, in Diário de Lisboa, 7, Julho, pp. 11-13.

\section{Interviews}

Viver Tóia, intervista al socio-fundatore di "Viver Troia. Associação dos proprietários pela defesa da qualidade urbana e ambiental", novembre 2017).

Tomás Taveira, architetto e collaboratore nell'Atelier Conceição Silva all'epoca della costruzione "Troia, Cidade Jardim". Intervista (aprile 2015) su "Troia, Cidade Jardim" e Bairro do Condado.

CML - Gebalis (Gestão do Arrendamento da Habitação Municipal de Lisboa), intervista alla presidente della Gebalis, ottobre 2016 e giugno 2017, ai tecnici responsabili della Zona J, settembre 2017.

Associação de residentes do Bairro do Condado, intervista al sócio responsabile, febbraio 2016. 